\title{
Coherent X-Ray Scattering by Phonons: Determination of Phonon Eigenvectors
}

\author{
H. Spalt and A. Zounek \\ Institut für Angewandte Physik, Technische Hochschule Darmstadt, D-6100 Darmstadt, \\ Federal Republic of Germany \\ and \\ B. N. Dev and G. Materlik \\ Hamburger Synchrotronstrahlungslabor (HASYLAB) at DESY, D-2000 Hamburg 52, Federal Republic of Germany
}

(Received 25 January 1988)

\begin{abstract}
Interference effects have been observed when coherently coupled $x$-ray beams are inelastically scattered by phonons in a crystal. The coherently coupled beams are prepared by dynamical diffraction methods. This principle was applied to determine the phases of the phonon eigenvectors in silicon by an analysis of the intensity of the inelastically scattered $x$ rays.

PACS numbers: $63.20 .-\mathrm{e}, 61.10 . \mathrm{Pa}, 78.70 . \mathrm{CK}$
\end{abstract}

The interatomic force constants-central to the theory of lattice dynamics of crystals-can be unambiguously determined experimentally by the measurement of both the frequency and the corresponding eigenvector of each phonon mode. ' Phonon frequencies as functions of wave vectors are obtained from inelastic neutron-scattering ${ }^{2}$ or $x$-ray-scattering ${ }^{3}$ experiments. In these conventional experiments, where the incident beam is describable by a single plane wave, the phonon eigenvectors cannot be directly determined because the scattered intensity is proportional to the square of the modulus of the dynamical structure factor $f_{\sigma}(\mathbf{K})$, which is complex in general and contains the eigenvectors. Thus the phase of $f_{\sigma}(\mathbf{K})$ and consequently the phases of the eigenvectors cannot be retrieved.

In this Letter we demonstrate a method for the solution of this phase problem via the observation of coherent scattering by phonons. The coherence arises from the use of probe beam which, instead of being a plane wave, is a coherent superposition of two plane waves of equal energies, and momenta differing only in direction but not in magnitude. In order to observe coherent scattering by phonons, we have (i) prepared the incident beam of coherently coupled plane waves, (ii) set the detector to detect $x$ rays scattered from the sample crystal by the phonons of a particular wave vector $\mathbf{q}$, and (iii) measured the intensity of the scattered radiation as a function of the relative phase of the plane-wave components in the incident beam. The phase dependence of the intensity is the manifestation of coherent scattering by phonons and can be used to determine the phonon eigenvectors.

A silicon sample crystal, cut parallel to the (111) planes, was used. This crystal itself generated the incident probe beam of two coherently coupled plane-wave photon states when the Bragg condition for a (111) reflection was satisfied. For a perfect crystal the incident and the Bragg-diffracted beam differ in momentum by a reciprocal-lattice vector, overlap in space, are coherently related, and are usually of similar strengths. The resulting wave field inside the crystal is of standing-wave nature, the properties of which are given by the dynamical theory of diffraction. ${ }^{4}$ The phase tunability of the diffracted beam and the associated movement of the standing-wave antinodal position with an external control of the angle of incidence or the energy of the incident $\mathrm{x}$ rays have been successfully used to locate atoms inside the crystal ${ }^{5,6}$ and on crystal surfaces. ${ }^{7,8}$ These properties have also been exploited in recent experiments to observe coherent Compton scattering. ${ }^{9}$

The present experiments to observe coherent photon scattering by phonons were performed at the ROEMO station of the Hamburg Synchrotron Laboratory HASYLAB at DESY in Hamburg with the storage ring DORIS as synchrotron radiation source. A sketch of the experimental arrangement, along with a reciprocal space representation, is shown in Fig. 1. The first Ge(111) monochromator crystal selected the desired energy from the continuous synchrotron radiation spectrum. The asymmetrically cut $\mathrm{Si}(111)$ second crystal was used to collimate the exit beam to an angular divergence of $\frac{1}{3}$ of the $\mathrm{Si}(111)$ natural reflection width [the slightly dispersive $\mathrm{Ge}(111)-\mathrm{Si}(111)$ arrangement helps additionally to suppress harmonics]. This monochromatized and collimated beam, a quasiplane wave, was directed towards a symmetric $\mathrm{Si}(111)$ crystal from which scattering of $\mathrm{x}$ rays by phonons was to be studied. The crystal was set at the Bragg angle $\left(\theta_{\mathrm{B}}=9.09^{\circ}\right)$ corresponding to an incident energy of $12.5 \mathrm{keV}$. The $\mathrm{NaI}$ detector was used to monitor the elastically scattered (diffracted) beam and the $\mathrm{Si}(\mathrm{Li})$ detector to detect the $\mathrm{x}$ rays inelastically scattered by the phonons. The $\mathrm{Si}(\mathrm{Li})$ detector resolved the Compton-scattered and the phonon-scattered peaks. The method of controlling and stabilizing the angle of in- 


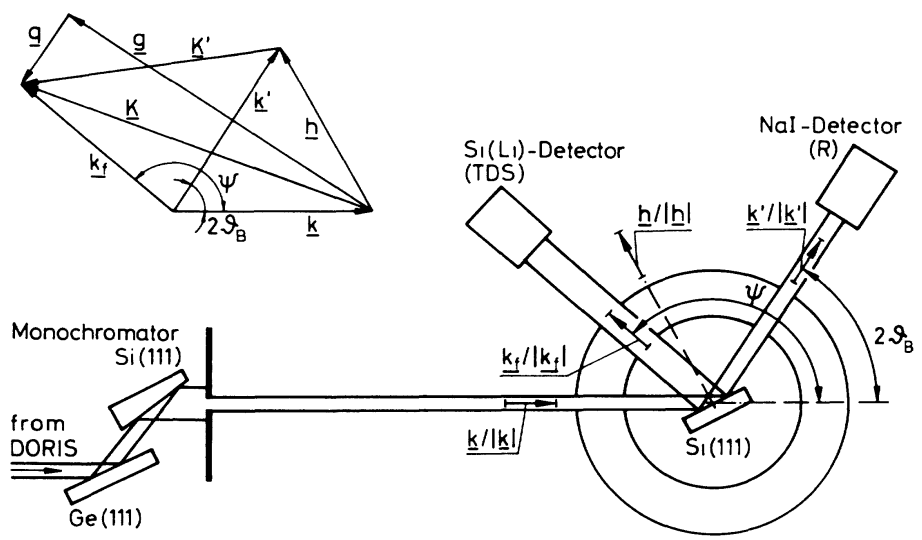

FIG. 1. Schematic layout of the experimental setup at the ROEMO station in HASYLAB and the k-space representation of the scattering geometry.

cidence has been described elsewhere. ${ }^{10}$ The accepted divergences of the inelastically scattered radiation were restricted to below $3^{\circ}$ in the plane of diffraction and $1^{\circ}$ in the plane perpendicular to it. The restriction on the divergence is to obtain a final state with a well-defined momentum and to ensure that scattering by phonons from a single Brillouin zone is detected. The k-space representation of the scattering is shown in the inset in Fig. 1, where $\mathbf{h}$ is the reciprocal lattice vector corresponding to (111) Bragg reflection, $\mathbf{k}$ and $\mathbf{k}^{\prime}=\mathbf{k}+\mathbf{h}$ are the wave vectors of the incident and the diffracted beam, respectively, and $\mathbf{k}_{f}$ is the wave vector of the inelastically scattered $x$ rays. $K=\mathbf{k}_{f}-\mathbf{k}$ and $K^{\prime}=\mathbf{k}_{f}-\mathbf{k}^{\prime}=\mathbf{K}-\mathbf{h}$ are the scattering vectors corresponding to the incident and Bragg diffracted beams in the crystal, respectively. $\mathbf{g}$ is the reciprocal lattice vector defining the phonon wave vector $\mathbf{q}$. In the absence of coherence the detected intensity is the sum of the intensities arising from the two beams of wave vectors $\mathbf{k}$ and $\mathbf{k}^{\prime}$, that is, corresponding to the scattering vectors $\mathbf{K}$ and $\mathbf{K}^{\prime}$, respectively. However, for coherently coupled beams we expect the scattered photon intensity to be the sum of the individual intensities arising from each beam plus interference effects in those final states accessible from any of the incident component states.

The results of measurements for two different phonon wave vectors $q$ around different reciprocal-lattice points are shown in Fig. 2. Figure 2(a) shows the reflectivity $(R)$ and the intensities of the inelastically scattered (thermal diffuse scattering, TDS) $\mathrm{x}$ rays as functions of angle of incidence. Contributions to the TDS curve from the incident beam (k), incident (k) plus diffracted $\left(\mathbf{k}^{\prime}\right)$ beams, and incident plus diffracted beams plus interference are shown by curves 1,2 , and 3 , respectively. In the strong reflection region the penetration of the incident beam into the crystal is greatly reduced compared to the angle of incidence outside the region. This effect is responsible for the reduced scattering yield at the middle of the TDS curves. The difference in the shapes of the TDS curves in Figs. 2(a) and 2(b) is basically due to differences in the strengths of the interference term that depends on the phase relations between the two scattering amplitudes. The variation of interference strength for individual types of phonons-LA, TA, LO, and TO-in a single group (a particular $\mathbf{g}$ and $\mathbf{q}$ ) is also reflected in the dissimilar asymmetry in their contributions to the total intensity [Fig. 2(b)]. (Some gross features of TDS associated with Bragg diffraction have been studied by Annaka. ${ }^{11}$ However, as a result of poor resolution of the detector, the contribution of Compton scattering was not separable from that of TDS. Moreover, because of the allowance of a large divergence of the scattered beam, the scattered radiation contained contributions of a wide range of phonons from several Brillouin zones.)

Having observed coherence in the $\mathrm{x}$ rays inelastically scattered by phonons, one may proceed for the extraction of the phases of the phonon eigenvectors. A method has recently been proposed by Kohl ${ }^{12}$ for the determination of phonon eigenvectors from coherent inelastic neutron scattering. We adapt this formalism to the case of coherent inelastic $x$-ray scattering and obtain, for the phonons of wave vector $q$ in the vicinity of the reciprocal-lattice point defined by $\mathbf{g}$, the differential cross section [from Eq. (B5) of Ref. 12],

$$
d \sigma / d \Omega=C \sum_{\sigma} \tau\left\{\left|f_{\sigma}(\mathbf{K})\right|^{2}+\left|E_{h} / E_{0}\right|^{2}\left|f_{\sigma}\left(\mathbf{K}^{\prime}\right)\right|^{2}+2 \operatorname{Re}\left[\left(E_{h} / E_{0}\right)^{*} f_{\sigma}(\mathbf{K}) f_{\sigma}^{*}\left(\mathbf{K}^{\prime}\right)\right]\right\}\left(2 \bar{n}_{\mathbf{q} \sigma}+1\right) / \omega_{\mathbf{q} \sigma},
$$

where

$$
f_{\sigma}(\mathbf{K})=\sum_{\mu} f_{\mu} M_{\mu}^{-1 / 2}\left(\mathbf{K} \cdot \mathbf{e}_{\mathbf{q} \sigma}^{\mu}\right) \exp \left(2 \pi i \mathbf{K} \cdot \mathbf{R}_{\mu}\right) \exp \left\{-W_{\mu}(\mathbf{K})\right\}
$$



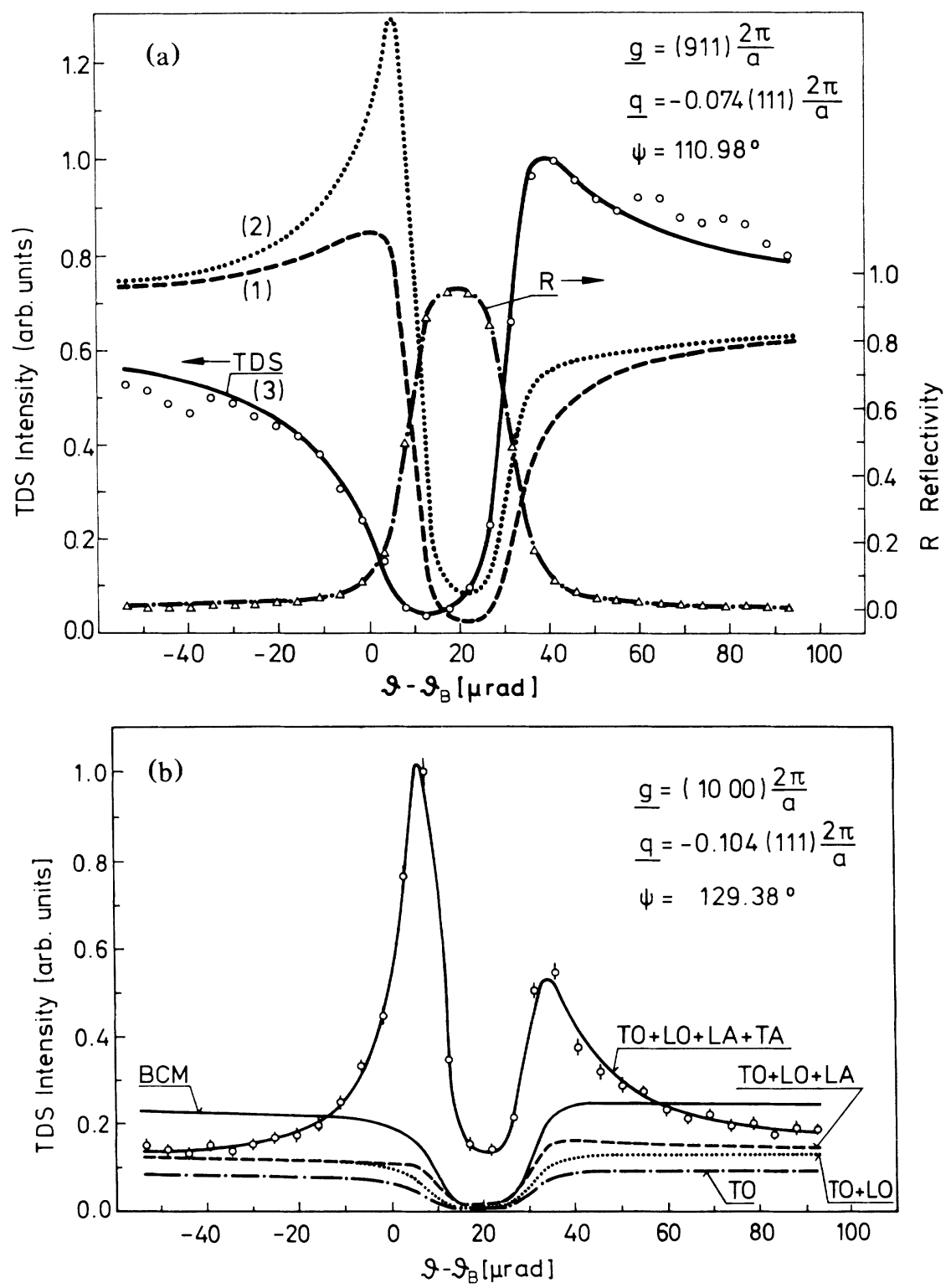

FIG. 2. (a) Measured reflectivity (triangles) for silicon (111) reflection and the intensity (open circles) of the $\mathrm{x}$ rays scattered by phonons near the (911) reciprocal lattice point as a function of the angle of incidence. The corresponding convoluted theoretical functions are shown by the dash-dotted and the solid lines. Curves 1, 2, and 3 represent the contributions of the first, first plus second, and first plus second plus third terms in Eq. (1), respectively. (b) Measured (open circles) and calculated (solid line) intensities of $\mathrm{x}$ rays scattered by phonons near the reciprocal-lattice point $(1000)$. The partial contributions from different branches of phonons are also shown. The curve marked " $\mathrm{BCM}$ " is obtained from the adiabatic bond-charge model as explained in the text.

is the dynamical structure factor or the scattering amplitude, which contains the phonon eigenvector $\mathbf{e}_{\mathbf{q} \sigma}^{\mu}$ of the mode $\mathbf{q} \sigma$. The eigenvector $\mathbf{e}_{\mathbf{q} \sigma}^{\mu}$ in turn contains the phase factor $\exp (i \delta)$, which determines the relative phase of the motion of the particles in the unit cell and is dependent on the interatomic force constants.

In Eq. (1), $C$ is a constant which also contains the number of atoms per unit cell. $\tau$ is the effective penetration depth and is proportional to the number of unit cells involved in the scattering. $E_{h} / E_{0}$ is the ratio of the electric field amplitude of the diffracted beam to that of the incident beam. This ratio is calculated as a function of angle of incidence from the theory of dynamical diffraction of $\mathrm{x}$ rays. $\left|E_{h} / E_{0}\right|^{2}$ is the reflectivity. The phase of $E_{h} / E_{0}$ is controlled experimentally by our changing the angle of incidence. Re represents the real part of the quantity in the square bracket. $\bar{n}_{\mathbf{q} \sigma}$ is the mean occupation number of the phonon mode $\mathbf{q} \sigma$ at the 
sample temperature and is given by the Bose-Einstein statistics. $\omega_{\mathbf{q} \sigma}$ is the phonon frequency of the mode $\mathbf{q} \sigma$. The summation runs over all the phonon branches. The scattering vectors $\mathbf{K}$ and $\mathbf{K}^{\prime}$ have been defined previously and are shown in the inset of Fig. 1. In Eq. (2) $f_{\mu}, M_{\mu}$, $\mathbf{R}_{\mu}$, and $W_{\mu}$ are the atomic scattering factor, the mass, the position, and the Debye-Waller exponent of the $\mu$ th atom within the unit cell. $\mathbf{e}_{\mathbf{q} \sigma}^{\mu}$ is the phonon eigenvector of the mode $q \sigma$. (Theoretical descriptions of TDS have been presented by Annaka, ${ }^{11}$ and Afanas'ev and Azizian. ${ }^{13}$ In the oversimplified treatment of TDS by Annaka the importance of the phase relations between the scattering amplitudes has not been considered, while in the work of Ref. 13, besides its ignoring the contributions of the optical phonons, the phonon characteristics have not been explicitly included.)

For an angle of incidence far away from the Bragg angle $\left(\theta_{\mathrm{B}}\right)$, the reflectivity is zero [see Fig. 2(a)], that is $E_{h} / E_{0}$ is zero. In this situation the second and the third terms in Eq. (1) vanish and we obtain the usual cross section for the scattering of a single beam (k). As we enter the strong reflection region, in addition to the contribution of the first term, the second and the third terms begin to contribute. The second term represents the cross section for the scattering of the second beam $\left(\mathbf{k}^{\prime}\right)$. The third term accounts for the interference effects between the two scattered waves with different directions of incidence. This interference term offers the possibility of extracting the phase of $f_{\sigma}(\mathbf{K})$, and consequently the phase of the eigenvector $\mathbf{e}_{\mathbf{q} \sigma}^{\mu}$.

The scattering cross sections were calculated with experimental phonon frequencies by Bilz and Kress ${ }^{14}$ and the theoretical eigenvectors with undetermined phases by Pope. ${ }^{15}$ The phase factors $\exp (i \chi)$ and $\exp (i \Theta)$ in the eigenvectors in the $\langle 111\rangle$ direction for the transverse (TA, TO) and longitudinal (LA, LO) phonons, ${ }^{15}$ respectively, determine the relative phase of the motion of the two particles in the diamond-lattice unit cell. The phases $\chi$ and $\Theta$ for silicon have been obtained by a nonlinear least-squares fit of the calculated intensity to the measured ones, and found to be $\chi=(155 \pm 10)^{\circ}$ and $\theta$ $=(165 \pm 25)^{\circ}$ for $\mathrm{q} \leq 0.13(2 \pi / a)$. For larger values of $\mathbf{q}$ the one-phonon theory does not provide a good fit and the contribution of two-phonon scattering ${ }^{16}$ to the measured intensities has presumably to be considered in more detail. A detailed report involving (111) and (100) phonons for different $\mathbf{q}$ values will be published elsewhere.

The eigenvectors given by Pope ${ }^{15}$ are based on Bornvon Kármán theory taking interactions between each atom and all its neighbors out to the sixth into account. We have also calculated the eigenvectors that contain no undetermined phases by the computer code of Nielsen and Weber ${ }^{17}$ which is based on the adiabatic bondchange (BCM) model. These eigenvectors showed poor agreement in most cases. One example is shown in Fig. 2(b), where under the experimental conditions the inelastic-scattering cross section is very sensitive to the phases of the eigenvectors. However, for a rigorous test of the BCM model, more experimental investigations would be necessary.

In conclusion, we have observed strong interference effects in the inelastic scattering of $x$ rays of coherently coupled incident states by phonons. The strength of the interference depends on the scattering conditions. It has been demonstrated that the phases of the phonon eigenvectors, that is, the relative phase of the motion of the ions in the unit cell, can be determined by quantitative analyses of the scattered intensities.

${ }^{1}$ R. S. Leigh, B. Szigeti, and V. K. Tewary, Proc. Phys. Soc. London A 320, 505 (1971); W. Cochran, Acta Crystallogr. Sect. A 27, 556 (1971).

${ }^{2}$ B. N. Brockhouse and P. K. Iyengar, Phys. Rev. 111, 747 (1957).

${ }^{3}$ C. B. Walker, Phys. Rev. 103, 547 (1956); for a direct measurement of inelastic scattering: B. Dorner, E. Burkel, Th. Illini, and J. Peisl, Z. Phys. B 69, 179 (1987).

${ }^{4} Z$. G. Pinsker, Dynamical Scattering of X-Rays in Crystals (Springer-Verlag, New York, 1978).

${ }^{5}$ B. W. Batterman, Phys. Rev. Lett. 22, 703 (1969).

${ }^{6}$ M. J. Bedzyk and G. Materlik, Phys. Rev. B 32, 6456 (1985).

${ }^{7}$ P. L. Cowan, J. A. Golovchenko, and M. F. Robbins, Phys. Rev. Lett. 44, 1680 (1980).

${ }^{8}$ G. Materlik, A. Frahm, and M. J. Bedzyk, Phys. Rev. Lett. 52, 441 (1984).

${ }^{9}$ J. A. Golovchenko, D. R. Kaplan, B. Kincaid, R. Levesque, A. Mexiner, M. F. Robbins, and J. Felsteiner, Phys. Rev. Lett. 46, 1454 (1981); W. Schülke, U. Bonse, and S. Mourikis, Phys. Rev. Lett. 47, 1209 (1981).

${ }^{10}$ A. Krolzig, G. Materlik, and J. Zegenhagen, Nucl. Instrum. Methods 208, 613 (1983); A. Krolzig, G. Materlik, M. Swars, and J. Zegenhagen, Nucl. Instrum. Methods Phys. Res. 219, 430 (1984).

${ }^{1}$ 'S. Annaka, S. Kikuta, and K. Kohra, J. Phys. Soc. Jpn. 21, 1559 (1966); S. Annaka, J. Phys. Soc. Jpn. 24, 1332 (1968).

${ }^{12}$ H. Kohl, Phys. Status Solidi (b) 130, 151 (1985).

${ }^{13}$ A. M. Afanas'ev and S. L. Azizian, Acta Crystallogr. Sect. A 37, 125 (1981).

${ }^{14} \mathrm{H}$. Bliz and W. Kress, Phonon Dispersion Relations in Insulators (Springer-Verlag, New York, 1979), p. 97.

${ }^{15}$ N. K. Pope, in Lattice Dynamics, edited by R. F. Wallis (Pergamon, Oxford, 1965), p. 147.

16J. S. Reid, Acta Crystallogr. Sect. A 41, 517 (1985).

${ }^{17}$ O. H. Nielsen and W. Weber, Comput. Phys. Commun. 18, 101 (1979). 